\title{
Incidentes relacionados a medicamentos em uma instituição hospitalar: subsídios para a melhoria da gestão
}

\section{Drug-related incidents in a hospital: input to improving management}

\author{
Incidentes relacionados a medicamentos en una institución hospitalaria: subsidios para la \\ mejoría de la gestión
}

\author{
Flávia Fernanda Rosa D’AquinoI; Carmen Maria Casquel Monti Julianil'; Silvana Andrea Molina Lima ${ }^{\text {III; }}$ \\ Wilza Carla Spiri ${ }^{\text {V }}$; Carmen Silva Gabrielv
}

\begin{abstract}
RESUMO: Estudo descritivo, retrospectivo e quantitativo, cujo objetivo é identificar os incidentes relacionados a medicamentos e seus fatores determinantes descritos em notificações espontâneas, em um hospital público do Estado de São Paulo. Os incidentes foram identificados a partir de 189 notificações espontâneas enviadas ao Núcleo de Segurança do Paciente, no período de junho de 2011 a junho de 2012. A taxa média de notificacões por 1000 pacientes-dia ao mês foi de 1,94. Os erros de medicação foram os incidentes mais notificados. A categoria de erro, classe medicamentosa, principais falhas e causas prováveis foram apresentadas. Do total dos incidentes, 19,8\% não causaram danos aos pacientes, 8,6\% causaram danos (eventos adversos) e 60,4\% deles foram interceptados pela enfermagem antes de atingir os pacientes (potencial evento adverso). Os resultados deste estudo fornecem subsídios aos profissionais envolvidos no sistema de medicamentos para a implantação de estratégias para a prevenção de incidentes. Palavras-Chave: Erros de medicação; sistemas de medicação no hospital; gerenciamento de segurança; segurança do paciente.
\end{abstract}

\begin{abstract}
This retrospective, quantitative, descriptive study to identify drug-related incidents and their determinants described in spontaneous reports at a hospital in São Paulo State. The incidents were identified from 189 spontaneous reports filed with the Patient Safety Center from June 2011 to June 2012. The average rate of notifications per 1000 patient-days per month was 1.94. Medication errors were the incidents most reported. Error category, medication type, main failures and likely causes were recorded. Of total incidents, 19.8\% caused no harm to patients, $8.6 \%$ caused harm (adverse events), and $60.4 \%$ were intercepted by nurses before affecting patients (potential adverse event). The results of this study provide input for professionals involved in the medication system to implement incident prevention strategies.

Keywords: Medication errors; hospital medication systems; safety management; patient safety.
\end{abstract}

RESUMEN: Estudio descriptivo, retrospectivo y cuantitativo cuyo objetivo es identificar los incidentes relacionados a medicamentos y sus factores determinantes descritos en informes espontáneos en un hospital del Estado de São Paulo. Los incidentes fueron identificados sobre 189 notificaciones espontáneas enviadas al Centro para Seguridad del Paciente, entre junio de 2011 y junio de 2012. La tasa media de notificaciones por 1000 pacientes-día al mes fue de 1,94. Los errores de medicación fueron los incidentes más reportados. Se presentaron la categoría de error, la clase medicamentosa, las principales fallas y las causas probables. Del total de incidentes, el 19,8\% no causó daño a los pacientes, un $8,6 \%$ causó daños (efectos adversos) y el $60,4 \%$ de errores fue interceptado por el personal de enfermería antes de afectar a los pacientes (potencial efecto adverso). Los resultados de este estudio proporcionan subsidios a los profesionales involucrados en el sistema de medicamentos para la puesta en marcha de estrategias para la prevención de incidentes.

Palabras Clave: Errores de medicación; sistemas de medicación en el hospital; gestión de seguridad; seguridad del paciente.

\section{INTRODUÇÃO}

A ocorrência de incidentes que afetam a segurança dos pacientes é uma preocupação mundial. A Organização Mundial de Saúde (OMS) lançou, em 2004, o Programa Aliança Mundial para a Segurança do Paciente ${ }^{1}$, que convoca os países a adotar estratégias para a melhoria da qualidade e assistência dos pacientes.

No Brasil, o Programa Nacional de Segurança do Paciente foi instituído pela Portaria $n^{\circ}$ 529/2013 do Ministério da Saúde, com objetivo de contribuir para a qualificação do cuidado em saúde ${ }^{2}$ e, consequentemente, prevenir os incidentes. Nesse mesmo ano, a Agência Nacional de Vigilância Sanitária (ANVISA) publicou a RDC 36/2013, que instituiu as ações para promoção da segurança do paciente e a melhoria da qualidade dos serviços de saúde ${ }^{3}$.

Entre os incidentes que afetam a segurança dos pacientes, os incidentes relacionados aos medicamentos ${ }^{\mathrm{VI}}$ (IRM) merecem atenção especial das instituições hospi-

IMestre em Enfermagem. Enfermeira do Instituto Lauro de Souza Lima. Bauru, São Paulo, Brasil. E-mail: flaviarosa_enf@yahoo.com.br.

IIDoutora em Enfermagem. Docente do Departamento de Enfermagem da Faculdade de Medicina de Botucatu. São Paulo, Brasil. E-mail: cjuliani@fmb.unesp.br. IIIDoutora em Enfermagem. Docente do Departamento de Enfermagem da Faculdade de Medicina de Botucatu. São Paulo, Brasil. E-mail: smolina@ fmb.unesp.br.

IV Doutora em Enfermagem. Docente do Departamento de Enfermagem da Faculdade de Medicina de Botucatu. São Paulo, Brasil. E-mail: wilza@fmb.unesp.br. vDoutora em Enfermagem. Docente da Escola de Enfermagem de Ribeirão Preto da Universidade de São Paulo. Brasil. E-mail: cgabriel@eerp.usp.br.

${ }^{\mathrm{VI}}$ Artigo decorrente da Dissertação do Mestrado Profissional Incidentes relacionados a medicamentos em uma instituição hospitalar: subsídios para a gestão. Autora

Flávia Fernanda Rosa D’Aquino, Faculdade de Medicina de Botucatu, 2014. 
talares, pois estudos epidemiológicos nos Estados Unidos estimam que cada paciente internado esteja sujeito a um erro de medicação por dia e, aproximadamente, 400.000 eventos adversos relacionados a medicamentos aconteçam por ano ${ }^{4}$.

Para a promoção de práticas seguras no uso de medicamentos e prevenção dos IRM, o Ministério da Saúde criou um protocolo de segurança na prescrição, uso e administração de medicamentos contendo estratégias para o monitoramento dos incidentes ${ }^{5}$.

O monitoramento dos incidentes pode ser realizado pela notificação espontânea dos envolvidos ${ }^{5}$, que é imprescindível para a melhoria da segurança dos pacientes, pois o seu conhecimento leva à avaliação dos processos de trabalho a fim de torná-los mais seguros.

Este artigo objetivou identificar os IRM e seus fatores determinantes nas notificações enviadas ao Núcleo de Segurança do Paciente (NSP) de um hospital público do Estado de São Paulo.

\section{REVISÃO DE LITERATURA}

Os incidentes são definidos como circunstâncias que poderiam ter resultado ou resultaram em danos desnecessários ao paciente ${ }^{6}$ e podem ser classificados como incidentes sem dano (incidente atingiu o paciente, mas não causou dano), incidentes com dano (evento adverso) ou near miss (incidentes interceptados antes de atingir o paciente), considerado um potencial evento adverso ${ }^{6,7}$.

Os incidentes relacionados a medicamentos (IRM) são quaisquer irregularidades no processo de uso de um medicamento e podem ocorrer em qualquer etapa do sistema de medicamentos ${ }^{8}$. Os enfermeiros são responsáveis legais pelo preparo e administração de medicamentos, etapa final desse complexo sistema que, se susceptível a falhas, pode determinar a ocorrência dos IRM ${ }^{9,10}$.

O enfermeiro, por sua função, tem a possibilidade de identificar falhas no preparo e administração de medicamentos e, com a ajuda de outros profissionais, conhecer as falhas em todo o sistema de medicamentos para, juntos, buscarem a prevenção dos incidentes.

\section{Metodologia}

Trata-se de estudo descritivo, retrospectivo com abordagem quantitativa que buscou os incidentes ocorridos na terapia medicamentosa de um hospital público do Estado de São Paulo.

O local do estudo é considerado um hospital de ensino, com 318 leitos e média de 7.346 pacientes-dia ao mês, Acreditado Nivel I pela Organização Nacional de Acreditação (ONA), faz parte da Rede Sentinela e é um serviço de referência regional. Possui oito unidades de internação, uma unidade de emergência, quatro unidades de terapia intensiva (adulto, pediátrica, queimados e coronariana), unidade de diagnóstico e terapia, três centros cirúrgicos, uma central de materiais e esterilização e um centro de terapia renal substitutiva.

Foram utilizadas todas as fichas de notificações espontâneas encaminhadas ao NSP no período de junho de 2011 a junho de 2012, totalizando-se 189 fichas. O NSP é um setor, na instituição, que tem como objetivo a análise de incidentes notificados e das causas e a mitigação dos incidentes, por meio da adoção de estratégias/barreiras, para preveni-los.

A coleta de dados foi realizada pelo pesquisador responsável, no primeiro semestre de 2013, por meio do acesso ao sistema informatizado de notificações espontâneas e das fichas de investigação dos incidentes utilizadas pelo NSP dessa instituição.

Os dados foram tabulados utilizando planilha Excel e submetidos à análise estatística descritiva em frequências e porcentagens, utilizando-se o programa SAS for Windows 9.2. A pesquisa foi aprovada pelo Comitê de Ética em Pesquisa (CEP), conforme ofício n ${ }^{\circ}$ 493/2012-CEP-Botucatu. Os procedimentos éticos foram seguidos, conforme a Resolução n 466/2012.

Os grupos farmacológicos foram registrados segundo a classificação Anatomical Therapeutic Chemical (ATC), isto é, classificação das drogas de acordo com o sistema anatômico, terapêutico e químico da World Health Organization (WHO) - Collaborating Centre for Drug Statistics Methodology ${ }^{11}$ e conforme a lista dos medicamentos de alto risco do Institute for Safe Medication Practices (ISMP) ${ }^{12}$, os quais, pela Agência Nacional de Vigilância Sanitária (ANVISA), são chamados de medicamentos potencialmente perigosos (MPP).

\section{Resultados e Discussão}

No total, foram enviadas 189 notificações ao NSP, o que representou uma taxa média de notificações por 1000 pacientes-dia ao mês de 1,94.

Outro estudo analisou os erros de medicação em um hospital pediátrico e apresentou uma taxa de 1,15 por 1000 pacientes-dia ${ }^{13}$. Apesar da taxa deste estudo ser expressiva em relação à literatura, a comparação fica limitada devido às diferentes características entre os hospitais. Porém, pode-se considerar que este estudo teve uma baixa frequência de notificações por ser um hospital de alta complexidade, pelo número de leitos, por ter quatro unidades de terapia intensiva, ser um hospital acreditado e participar da Rede de Hospitais Sentinela, o que poderia estimular as notificações.

Entre os IRM, os erros de medicação foram os mais identificados - 116(61,5\%), seguidos das reações adversas a medicamentos - 40(21,2\%), queixas técnicas - 22(11,7\%) e inefetividade terapêutica - 11(5,6\%). Outros estudos também apontaram os problemas na terapia medicamentosa como sendo os mais notificados $-63 \%{ }^{14}$ e $71 \%{ }^{15}$.

Em relação à origem das notificações, as unidades que mais notificaram foram as de clínica médica $49(25,9 \%)$ e pediatria 35(18,5\%). As frequências das notificações por unidade estão dispostas na Tabela 1. 
TABELA 1: Frequências das notificações dos incidentes relacionados a medicamentos por unidade procedente. Bauru, SP, junho de 2011 a junho de 2012

\begin{tabular}{lcc}
\hline \multicolumn{1}{c}{ Unidades } & \multicolumn{2}{c}{ Notificações } \\
& $\mathrm{f}$ & $\%$ \\
\hline Clínica médica & 49 & 25,9 \\
Pediatria & 35 & 18,5 \\
Centro de diagnóstico e imagem & 25 & 13,2 \\
Unidade coronariana & 14 & 7,4 \\
Clínica cirúrgica & 12 & 6,3 \\
Emergência & 10 & 5,3 \\
Unidade de terapia intensiva pediátrica & 7 & 3,7 \\
Unidade de terapia intensiva adulto & 6 & 3,2 \\
Centro cirúrgico & 5 & 2,6 \\
Quimioterapia & 5 & 2,6 \\
Unidade de queimados & 3 & 1,6 \\
Ressonância magnética & 3 & 1,6 \\
Acolhimento & 2 & 1,1 \\
Centro de terapia renal substitutiva & 2 & 1,1 \\
Oncologia & 2 & 1,1 \\
Unidade de terapia intensiva coronariana & 2 & 1,1 \\
Outras unidades ${ }^{*}$ ) & 7 & 3,7 \\
Total & 189 & 100 \\
\hline
\end{tabular}

Fonte: Fichas de notificação e investigação.

(*) Outras unidades que tiveram uma notificação: farmácia, farmácia de alto custo, centro cirúrgico, farmácia de oncologia, endoscopia, centro cirúrgico ambulatorial e ambulatório.

Em estudo de notificações espontâneas, a unidade que mais notificou também foi a clínica médica ${ }^{14}$. As ocorrências nas unidades de pediatria são esperadas, pois os pacientes pediátricos estão três vezes mais predispostos a erros de medicação e eventos adversos relacionados a medicamentos em relação à população adulta por diversos fatores, como as diferentes formas de dosagens do mesmo medicamento, variedades das concentrações das soluções, dosagem dependente do peso do paciente e de cálculos e também da própria maturidade dos órgãos que podem dificultar a metabolização e excreção dos medicamentos ${ }^{16}$.

A unidade que mais notifica não é necessariamente aquela que tem mais incidentes, pois a que tem menor número pode estar subnotificando. Assim, baixas frequências de notificação não significam sistemas seguros, podendo, inclusive, significar o contrário, ou seja, por se tratar de equipes mais preparadas e conscientes para a problemática, tendem a notificar mais.

A subnotificação é um aspecto importante a ser considerado nos serviços de saúde e, para sua redução, a notificação de ocorrências deve ser sempre orientada e incentivada por toda a equipe ${ }^{17}$. A subnotificação pode ocorrer por diversos fatores como medo, culpa e tipo de sistema de notificação, sendo uma das principais dificuldades do método de notificação voluntária ${ }^{15}$.

As categorias dos erros de medicação notificadas foram a dispensação errada - 56(48,3\%), paciente errado - 16(13,8\%), medicamento errado - 13(11,2\%), dose errada - 10(8,6\%), prescrição errada - 9(7,8\%), horário errado $-7(6 \%)$, via errada $-3(2,6 \%)$ e a omissão de dose -2(1,7\%). Em comparação com outros estudos, a categoria de erros de medicação mais presente foi a omissão de dose ${ }^{18,19}$, que não foi representativa neste estudo.

A classe medicamentosa mais envolvida nas notificações de erros de medicação foram os antibióticos sistêmicos, representando 22 (19\%) registros. Os erros na administração dos antibióticos podem resultar em resistência microbiana. Esta pesquisa corrobora estudo multicêntrico brasileiro que encontrou uma taxa de erros na administração de antimicrobianos de 18,5\% ${ }^{20}$.

Neste estudo, 63(54,3\%) notificações abrangeram classes medicamentosas que correspondem a medicamentos potencialmente perigosos (MPP). Por questão de classificação, três das notificações que envolviam antibióticos na forma lipossomal foram somados aos MPP. As características desses medicamentos estão apresentadas na Tabela 2.

Estudo brasileiro, que retratou os MPP, revelou

TABELA 2: Características dos medicamentos registrados nas notificações. Bauru, SP, junho de 2011 a junho de 2012.

\begin{tabular}{|c|c|c|}
\hline Características & $f$ & $\%$ \\
\hline \multicolumn{3}{|l|}{ Classificação Anatomical Therapeutic } \\
\hline \multicolumn{3}{|l|}{ Chemical } \\
\hline Antibióticos sistêmicos & 22 & 19,0 \\
\hline Soluções intravenosas e seus aditivos & 19 & 16,4 \\
\hline Analgésicos & 14 & 12,1 \\
\hline $\begin{array}{l}\text { Agonistas adrenérgicos, antagonistas } \\
\text { adrenérgicos, antiarrítmicos, } \\
\text { medicações específicas do sistema } \\
\text { cardiovascular (nitroprussiato de sódio) }\end{array}$ & 12 & 10,3 \\
\hline $\begin{array}{l}\text { Drogas usadas no diabetes (insulina e } \\
\text { hipoglicemiantes orais) }\end{array}$ & 5 & 4,3 \\
\hline Agentes antitrombóticos & 3 & 2,6 \\
\hline $\begin{array}{l}\text { Radiofármacos para diagnóstico } \\
\text { (contraste) }\end{array}$ & 3 & 2,6 \\
\hline Sedativos moderados & 3 & 2,6 \\
\hline Bloqueadores neuromusculares & 1 & 0,9 \\
\hline Ansiolíticos (benzodiazepínicos) & 5 & 4,3 \\
\hline $\begin{array}{l}\text { Inibidor da bomba de prótons, antiespas- } \\
\text { módico, antiemético e antinauseante }\end{array}$ & 5 & 4,3 \\
\hline Antiepiléticos & 2 & 1,7 \\
\hline Agentes modificadores dos lipídios & 2 & 1,7 \\
\hline Drogas para constipação - enemas & 2 & 1,7 \\
\hline Antimicobacteria & 2 & 1,7 \\
\hline \multirow[t]{2}{*}{ Outros $^{(*)}$} & 16 & 13,8 \\
\hline & 116 & 100 \\
\hline Medicamentos potencialmente perigosos ${ }^{(* *)}$ & 63 & 54,3 \\
\hline
\end{tabular}

Fonte: Fichas de notificação e investigação.

(*) Outros: antianêmico, anti-hemorrágico, anti-inflamatório, antiespasmódico urinário, hormônio tireoidiano, broncodilatador, antigotoso, antisséptico dermatológico, medicação para a Doença de Alzheimer, medicamentos sem identificação.

${ }^{(* *)}$ Inclusive três antibióticos na forma lipossomal. 
taxas de $37,4 \%{ }^{19}$. Todos os erros de medicação podem levar a danos aos pacientes, porém os MPP descritos pelo ISMP têm alto risco para danos e as consequências podem ser devastadoras ${ }^{12}$, o que torna estes achados preocupantes.

Dados de estudo realizado, no Brasil, com 14 gerentes de risco mostram que as iniciativas menos utilizadas para a segurança do paciente são as relacionadas ao controle de MPP e prevenção de eventos adversos com medicamentos ${ }^{21}$. O ISMP orienta que as instituições padronizem as etapas de ordenação, armazenamento, preparo e administração, melhorem $\mathrm{o}$ acesso à informação sobre os MPP, usem etiquetas auxiliares para identificação, alertas automáticos e dupla checagem quando necessária ${ }^{12}$.

As falhas de maior ocorrência foram a dispensação de medicamentos trocados - 28(24,1\%), dispensação de medicamento para paciente errado - 13(11,2\%) e administração do medicamento em paciente errado - 12(10,3\%), seguidos de dispensação de medicamento com volume que indica via errada - 10 (8,7\%), dispensação de medicamento com a dose errada $-9(7,8 \%)$, medicamento não dispensado - 7(6\%), dispensação de medicamento com o nome da etiqueta diferente do medicamento - 4(3,4\%), dispensação do medicamento com tampa não estéril - 1(0,9\%), dispensação de medicamento com corpo estranho entre o êmbolo e a seringa $-1(0,9 \%)$, dispensação de medicamento com a validade vencida $-1(0,9 \%)$, troca de nome nas fitas dos pacientes $-4(3,4 \%)$, medicamento administrado duas vezes $-2(1,7 \%)$, medicamento não administrado $-2(1,7 \%)$, administrado na dose errada $-5(4,3 \%)$, administrado no horário errado $-4(3,4 \%)$, administrado medicamento em via errada $-2(1,7 \%)$, prescrição de medicamento a paciente alérgico $-4(3,4 \%)$, prescrição de medicamento para a mãe na prescrição do paciente/ filho $-1(0,9 \%)$, prescrição em local errado (farmácia não consegue visualizar) $-1(0,9 \%)$, prescrição em dose errada $-1(0,9 \%)$, prescrição de medicamento não padronizado - $1(0,9 \%)$, impressão de prescrição médica antiga pela enfermagem $-2(1,7 \%)$, medicamento não dispensado na alta pela enfermagem $-1(0,9 \%)$.

A dispensação de medicamentos trocados estava relacionada a medicamentos com nomes e sons semelhantes, ampolas semelhantes e soluções de infusão e seus aditivos que foram enviados em concentrações diferentes das prescritas. Autoridades mundiais, indústrias farmacêuticas e as instituições devem dar maior importância a esses medicamentos como melhoria na segurança dos pacientes ${ }^{22}$.

Algumas estratégias são descritas pela OMS para a prevenção desses erros, como realizar levantamento anual dos medicamentos, realizar a orientação dos profissionais, minimizar solicitações por ordens verbais, dar importância à leitura dos rótulos e não somente seu reconhecimento visual e do lugar físico do medicamento, conhecer o efeito do medicamento, garantir a legibilidade da prescrição, incluindo o nome genérico e a forma farmacêutica do medicamento, esclarecer ordens verbais antes da administração, separar fisicamente os medicamentos com nomes semelhantes, utilizar a escrita com maiúsculas e minúsculas na prescrição desses medicamentos e orientar os pacientes sobre os seus medicamentos para que possam reconhecer problemas relacionados aos sons e nomes semelhantes em relação ao seu medicamento ${ }^{22}$.

Em relação à dispensação de medicamentos para pacientes errados, as orientações da OMS para minimizar esses erros são enfatizar a responsabilidade dos profissionais na identificação dos pacientes antes de qualquer procedimento, incentivar o uso de pelo menos dois identificadores (por exemplo: o nome e a data de nascimento), padronizar as abordagens para a identificação (uso de pulseiras, identificação a ser realizada pelo nome e data de nascimento ou com o uso de tecnologias), padronizar abordagens para pacientes de nomes semelhantes, encorajar os pacientes a participarem de todo o processo, incentivar a rotulagem dos recipientes utilizados, treinamento contínuo dos profissionais e encorajar os familiares a relatarem $\operatorname{erros}^{23}$.

As causas prováveis mais relatadas nas fichas de investigação foram a falha na dupla checagem dos medicamentos na farmácia - 31(26,7\%), a falha na adesão às diretrizes medicamentosas - 24(20,7\%) e a falha na comunicação - $10(8,6 \%)$, seguidas de falha na manipulação - $8(6,9 \%)$, sons e grafias semelhantes e frascos semelhantes - 5(4,3\%), farmácia seguiu o parecer do serviço de controle de infecção ao invés da prescrição médica $5(4,3 \%)$, problema na mão de obra da farmácia $-4(3,4 \%)$, mudanças de rotina na farmácia $-2(1,7 \%)$, problemas no processo de compra $-2(1,7 \%)$, prescrição à caneta $-1(0,9 \%)$, fita das medicações abertas simultaneamente e medicamentos trocados pela enfermagem - $1(0,9 \%)$, etiqueta de um medicamento grudou no outro-1 $(0,9 \%)$, comprimido não pode ser triturado, enviado em solução $-1(0,9 \%)$, troca de bombas de infusão $-1(0,9 \%)$, falha na investigação de alergia do paciente $-1(0,9 \%)$, desconhecimento dos perigos pelo médico (prescrição para a mãe na prescrição do paciente) $-1(0,9 \%)$, desconhecimento da dosagem pediátrica $-1(0,9 \%)$ e causa provável estava ausente nos registros - 17(14,5\%).

A dupla checagem no contexto deste estudo é uma atividade realizada por técnicos de farmácia, que são seres humanos e passíveis de $\operatorname{erros}^{24}$. Os sistemas automatizados podem auxiliar no controle da dispensação errada e diminuir os erros de medicação $0^{25}$.

Outra causa provável dos erros de medicação foi a falha na adesão às diretrizes institucionais. Estudo norte-americano também aponta o problema da não adesão às diretrizes medicamentosas, indicando a necessidade de diretrizes mais práticas para a administração de medicamentos, fator este relevante 
para o exercício clínico ${ }^{26}$. Conhecer as dificuldades de processo dos profissionais para a execução segura e planejar ações que facilitem a adesão às diretrizes devem ser ações institucionais permanentes.

A falha na comunicação foi a terceira causa provável dos erros de medicação descrita nas investigações deste estudo. $\mathrm{O}$ processo de comunicação no hospital é complexo devido ao número de profissionais e ao número de informações e atividades; as falhas representam a falta de processos integrados e envolve a transferência das informações e a aceitação da responsabilidade do cuidado ${ }^{27}$.

Em relação aos incidentes, $70(60,4 \%)$ foram interceptados pela enfermagem antes de atingir os pacientes (potencial evento adverso - near miss), 23(19,8\%) atingiram os pacientes, mas não causaram danos, intercorrências ou sinais vitais alterados (incidentes sem dano) e 10(8,6\%) causaram danos (eventos adversos). Em 13(11,2\%) notificações, os registros sobre os danos estavam ausentes. Estudo de seis anos na Inglaterra mostrou que a maioria dos incidentes não resultou em danos - 83\%, 13\% apresentaram danos leves, $0,15 \%$ ocasionaram danos severos e morte ${ }^{18}$.

Neste estudo, a enfermagem teve papel importantíssimo na interceptação de $60,4 \%$, dos IRM, porém, verifica-se a necessidade de fortalecer as etapas anteriores, pois sistemas seguros deveriam interceptar erros até mesmo antes da administração. A etapa da administração de medicamentos é considerada vulnerável e os erros são menos propensos de serem interceptados nessa fase $\mathrm{e}^{28}$, devido ao número de atividades realizadas, com exigência de vários protocolos e diretrizes institucionais ${ }^{29}$.

\section{ConClusão}

Este estudo permitiu identificar os IRM e seus fatores determinantes. Esforços conjuntos de profissionais do sistema de medicamentos devem ser direcionados para o objetivo de diminuir os incidentes e melhorar a cultura de segurança do paciente.

Embora os resultados demonstrem a importância da atuação da equipe de enfermagem, a qual interceptou $60,4 \%$ dos erros, este dado deve ser visto com preocupação, pois a equipe de enfermagem, que trabalha com sobrecarga de atividades nas instituições hospitalares, não deveria receber medicações em desacordo com as normas, pois tal condição aumenta o risco aos pacientes. $\mathrm{O}$ ideal é que a checagem anterior permitisse a dispensação e identificação corretas dos medicamentos.

\section{REFERÊNCIAS}

1.World Health Organization. World Alliance for Patient Safety: forward programme [site de Internet]. 2006-2007. Geneva (Swi). [cited in 2014 Jun 10] Disponível em: http://www.who.int/patientsafety/information_centre/
WHO_EIP_HDS_PSP_2006.1.pdf.

2.Ministério da Saúde (Br). Programa Nacional de Segurança do Paciente. Portaria n ${ }^{\circ} 529$ de $1^{\circ}$ de abril de 2013. Institui o Programa Nacional de Segurança do Paciente. Brasília (DF): Gabinete Ministerial; 2013.

3.Ministério da Saúde (Br). Resolução - RDC n ${ }^{\circ}$ 36, 25 de julho de 2013. Institui ações para a segurança do paciente em serviços de saúde e dá outras providências. Brasília (DF): Gabinete Ministerial; 2013.

4.Institute of Medicine. Preventing medication errors: quality chasm series. Washington (DC): Nacional Academy Press; 2006.

5.Ministério da Saúde $(\mathrm{Br})$. Protocolo de segurança na prescrição, uso e administração de medicamentos. Protocolo integrante do Programa Nacional de Segurança do Paciente. Brasília (DF): Ministério da Saúde; 2013.

6. World Health Organization. The conceptual framework for the international classification for patient safety. Version 1.1. [site Internet]. WHO; 2009. [cited in 2014 jun 10] Available from: http://www.who.int/patientsafety/ taxonomy/icps_chapter3.pdf.

7.Capucho HC. Near miss: quase erro ou potencial evento adverso? [Carta ao editor]. Rev Latino-Am Enfermagem. 2011;19:[2 telas]. [citado em jul 2014] Disponível em: http://www.scielo.br/pdf/rlae/v19n5/pt_27.pdf.

8.Morimoto T, Gandhi TK, Seger AC, Hsieh TC, Bates DW. Adverse drug events and medication errors: detection and classification methods. Qual Saf Health Care. 2004; 13:306-14.

9.Cassiani SHB, Miasso AL, Silva AEBC, Fakin FT, Oliveira $\mathrm{RC}$. Aspectos gerais e número de etapas do sistema de medicação de quatro hospitais brasileiros. Rev Latino-Am Enfermagem. 2004; 12:781-9.

10.Oliveira RB, Melo ECP. O sistema de medicação de um hospital especializado no Município do Rio de Janeiro. Esc Anna Nery. 2011; 15:480-9.

11.World Health Organization. Collaborating Centre for Drug Statistics Methodology, Guidelines for ATC classification and DDD assignment 2013 [site Internet]. Oslo, 2012. [cited in 2014 May 03] Available from: http://www. whocc.no/filearchive/publications/1_2013guidelines.pdf.

12.Institute for Safe Medication Practice. ISMP's list of higth-alert medications [site Internet]. [cited in $2014 \mathrm{Nov}$ 30] Available from: http://www.ismp.org/tools/highalertmedications.pdf.

13.Yamamoto MS, Peterlini MAS, Bohomol E. Notificação espontânea de erros de medicação em hospital universitário pediátrico. Acta Paul Enferm. 2011; 24:766-71.

14.Bezerra ALQ, Silva AEBC, Branquinho NCSS, Paranaguá TTB. Análise de queixas técnicas e eventos adversos em um hospital sentinela. Rev enferm UERJ. 2009; 17:467-72.

15.Capucho HC, Arnas ER, Cassiani SHB. Segurança do paciente: comparação entre notificações voluntárias manuscritas e informatizadas sobre incidentes em Saúde. Rev Gaúcha de Enferm. 2013;34:164-72.

16.Gonzales Kelly. Medications administration errors 
and the pediatric population: a systematic search of the literature. Journal of Pediatric Nursing. 2010; 25:555-65. 17.Lima PF, Cavassini ACM, Silva FAT, Kron MR, Gonçalves SF, Spadotto A, et al. Queixas técnicas e eventos adversos a medicamentos notificados em um hospital sentinela do interior de São Paulo, 2009-2010. Epidemiol Serv Saúde. 2013; 22:679-86.

18.Cousins DH, Gerrett D, Warner B. A review of medication incidents reported to the Nacional Reporting and Learning System in England and Wales over 6 years (2005-2010). Br J Clin Pharm. 2011; 47:597-604.

19.Silva AEBC, Reis AMM, Miasso AI, Santos JO, Cassiani SHB. Eventos adversos a medicamentos em um hospital sentinela do Estado de Goiás, Brasil. Rev Latino-Am Enfermagem. 2011; 19: [9 telas].

20.Marques TC, Reis AMM, Silva AEBC, Gimenes FRE, Opitz SP, Teixeira TCA et al. Erros de administração de antimicrobianos identificados em estudo multicêntrico brasileiro. Rev Bras Ciências Farm. 2008; 44:305-14.

21.Souza RFF, Silva LD. Estudo exploratório das iniciativas acerca da segurança do paciente em hospitais do Rio de Janeiro. Rev enferm UERJ. 2014; 22:22-8.

22. World Health Organization. Patient safety solutions. 2007 [site Internet]. [cited in 2014 Dec 07] Available from: http://www.who.int/patientsafety/solutions/patient-
safety/PS-Solution1.pdf.

23. World Health Organization. Patient safety solutions. 2007 [site Internet]. [cited in 07 Dec 2013] Available from: http://www.who.int/patientsafety/solutions/patientsafety/PS-Solution2.pdf.

24.Reason J. Human error: models and management. BMJ 2000; 320:768-70.

25.Chapuis C, Roustit M, Bal G, Schwebel C, Pansu P, Tchouda SD et al. Automated drug dispensing system reduces medication errors in an intensive care setting. Crit Care Med. 2010; 38:2275-81.

26.Kim J, Bates DW. Medication administration errors by nurses: adherence to guidelines. J Clin Nurse. 2012; 590-8. 27.Agência Nacional de Vigilância Sanitária (ANVISA). Assistência segura: uma reflexão teórica aplicada a prática [site de Internet]. Ministério da Saúde; 2013. [citado em 15 out 2013] Disponível em: http://www20. anvisa.gov.br/segurancadopaciente/images/documentos/ livros/Livro1-Assistencia_Segura.pdf.

28.Leape LL, Bates DW, Cullen DJ, Cooper J, Demonaco HJ, Gallivan T et al. Systems analysis of adverse drug events. JAMA.1995; 274:35-43.

29.Jennings BM, Sandelowski M, Mark B. The nurse's medication day. Qualitative Health Research. 2011; 21:1441-51. 